\title{
Sunitinib in Patients with Cisplatin-Refractory Germ Cell Tumors
}

\author{
Maria Reckova a,b,c Michal Mego ${ }^{b, c}$ Zuzana Sycova-Mila ${ }^{c}$ \\ Jana Obertova $^{\mathrm{b}, \mathrm{c}} \quad$ Dana Svetlovska $^{\mathrm{b}, \mathrm{C}}$ Jozef Mardiak ${ }^{\mathrm{b}, \mathrm{c}}$ \\ ancology Clinic Poprad, \\ ${ }^{b}$ Department of Medical Oncology School of Medicine, Comenius University, \\ 'National Cancer Institute, Bratislava, Slovakia
}

It was reported previously that monotherapy with sunitinib has limited efficacy in patients with heavily pretreated, unselected, refractory germ cell tumors $[1,2]$. However it is of great interest to explore the molecular characteristics of responding patients as it may allow identification of a molecular profile suitable for sunitinib therapy or treatment with other targeted agents [1].

At the National Cancer Institute of Slovakia, we performed a 1-arm, single-stage, phase II study aimed at evaluating the efficacy and toxicity of sunitinib in patients with refractory germ cell tumors. The study was multicentric, with participation of 3 study centers in Slovakia. The Ethical Committee of the National Cancer Institute in Bratislava approved the study which was registered in the European Clinical Trials Database, EudraCT number 2007-004981-42. Those patients with radiological and/or serological proof of relapsed metastatic germ cell tumors, who were not eligible for curative chemotherapy or surgery and had failed at least 2 platinum-based regimens or 1 platinum regimen in the case of platinum-refractory disease or primary mediastinal nonseminomatous germ cell tumor, were eligible. All other standard inclusion and exclusion criteria for the study of salvage treatment in refractory germ cell tumor patients were applied. We planned to study 28 patients. However due to poor accrual, only 10 patients were included in the trial (July 2008 to December 2010). All patients gave informed consent. The median age was 32 years (range 19-55 years). All patients were platinum-refractory, with the exception of one. Patients were pretreated with a median of 3 cisplatin-containing therapies (range 3-6). Sunitinib was administered at a dose of $50 \mathrm{mg}$ daily for 4 weeks, followed by 2 weeks off therapy. Sunitinib had limited efficacy in this treatment population with a median progression-free survival and overall survival of 10.8 and 12.9 weeks, respectively. RECIST 1.0 criteria and/ or tumor markers were used to evaluate the response. Tumor markers were measured every 6 weeks, and computed tomography (CT) scans of the chest, abdomen, and pelvis were evaluated every 12 weeks. CT or magnetic resonance imaging studies of the brain and bone scans were carried out where clinically indicated. 1 patient with teratocarcinoma achieved partial remission in the lungs and retroperitoneal lymph nodes as well as a decrease in alpha-fetoprotein (AFP) after 2 treatment cycles, but progressed after 4 cycles. Another patient with a teratoma component in the primary tumor and high levels of beta-human chorionic gonadotropin (b-hCG) at relapse, achieved partial remission in the lungs and lymph nodes after 2 treatment cycles; however rapid elevation of b-hCG was seen after 4 cycles.

In the study reported by Oechsle et al. [1], 2 patients with non-seminoma achieved partial remission, however it is not clear if a teratoma component was present in these germ cell tumors. Several translational studies suggested that vascular endothelial growth factor (VEGF) might play an important role in the growth and progression of germ cell tumors $[3,4]$. Substantially higher VEGF expression has been found in patients with germ cell tumors compared with normal testis tissue. VEGF overexpression was most often seen in teratoma components of such tumors [3]. Teratoma is the most frequent histological element in late-relapsing germ cell tumors. Recently, Ramasubbaiah et al. [5] observed that 5 of 8 patients with late relapse responded to a combination of oxaliplatin and bevacizumab. However the histology of the responding patients was not reported. All patients that responded had rising serum hCG and/or AFP levels. Radiographic responses were observed at the time of declining markers, and thus it was impossible to know if any teratoma component was part of the response (personal communication with L.H. Einhorn). Previously, we observed the efficacy of bevacizumab in the treatment of growing teratoma syndrome [6].

\section{KARGER}

Fax +497614520714

Information@Karger.de

www.karger.com (c) 2012 S. Karger GmbH, Freiburg

0378-584X/12/0357-0455\$38.00/0

Accessible online at:

www.karger.com/onk
Maria Reckova, MD

Oncology Clinic Poprad

Mnohelova 2, 05801 Poprad, Slovaki

Tel. +421 52-4314168, Fax -7763137

maryrecka@gmail.com 
We are aware of the limitations of this small phase II study which yielded only hypothesis-generating results. However understanding these limitations and based on our observations, we conclude that sunitinib has limited efficacy in unselected refractory germ cell tumor patients with a possibility of some efficacy in a selected group of patients with teratoma. Further, properly conducted clinical trials with robust translational research are of great importance, and cooperation on a multinational level in designing such trials in patients with refractory germ cell tumors is greatly needed.

\section{Acknowledgement}

Special thanks to Stephen E. Zlatos Esq. for his assistance in the editing of the manuscript.

\section{Disclosure Statement}

This study was supported by Pfizer Luxembourg SARL, o.z. Slovakia. The authors declare no conflict of interest.

\section{References}

1 Oechsle K, Honecker F, Cheng T, et al.: Preclinical and clinical activity of sunitinib in patients with cisplatin-refractory or multiply relapsed germ cell tumors: a Canadian Urologic Oncology Group/German Testicular Cancer Study Group cooperative study. Ann Oncol 2011;22:2654-60.

2 Feldman DR, Turkula S, Ginsberg MS, et al.: Phase II trial of sunitinib in patients with relapsed or refractory germ cell tumors. Invest New Drugs 2010; 28:523-8.

3 Adam M, Schmidt D, Wardelmann E, et al.: Angiogenetic protooncogene ets-1 induced neovascularization is involved in the metastatic process of testicular germ cell tumors. Eur Urol 2003;44:329-36.

4 Fukuda S, Shirahama T, Imazono Y, et al.: Expression of vascular endothelial growth factor in patients with testicular germ cell tumors as an indicator of metastatic disease. Cancer 1999;85:1323-30.

5 Ramasubbaiah R, Brames J, Johnston EL, et al.: Phase II study of oxaliplatin (O) and bevacizumab (B) chemotherapy in refractory germ cell tumors (GCT). J Clin Oncol 2010;28:abstr e15054.

6 Mego M, Recková M, Sycova-Mila Z, et al.: Bevacizumab in a growing teratoma syndrome. Case report. Ann Oncol 2007;18:962-3. 